\title{
Multiplicity of Positive Solutions for a Second-Order Elliptic System of Kirchhoff Type
}

\author{
S. Khademloo, E. Valipour, and A. Babakhani \\ Department of Mathematics, Faculty of Basic Sciences, Babol Noshirvani University of Technology, Babol 4714871167, Iran \\ Correspondence should be addressed to S. Khademloo; s.khademloo@nit.ac.ir
}

Received 14 July 2014; Revised 18 August 2014; Accepted 25 September 2014; Published 12 November 2014

Academic Editor: Chuanzhi Bai

Copyright (C) 2014 S. Khademloo et al. This is an open access article distributed under the Creative Commons Attribution License, which permits unrestricted use, distribution, and reproduction in any medium, provided the original work is properly cited.

We study elliptic problems of Kirchhoff type in $\Omega \subset \mathbb{R}^{N}(N \geq 3)$. Using variational tools, we establish the existence of at least two nontrivial and nonnegative solutions.

\section{Introduction and Preliminaries}

In this paper, we are concerned with the following problem:

$$
\begin{gathered}
-\left(a_{1}+b_{1}\left(\int_{\Omega}|\nabla u|^{2}+a(x)|u|^{2}\right) d x\right)(\Delta u+a(x) u) \\
=F_{u}(x, u, v)+\lambda b(x)|u|^{q-2} u \quad \text { in } \Omega, \\
-\left(a_{2}+b_{2}\left(\int_{\Omega}|\nabla v|^{2}+a(x)|v|^{2}\right) d x\right)(\Delta v+a(x) v) \\
=F_{v}(x, u, v)+\mu c(x)|v|^{q-2} v \quad \text { in } \Omega, \\
u=v=0 \quad \text { on } \partial \Omega,
\end{gathered}
$$

where $\Omega \subset \mathbb{R}^{N}(N \geq 3)$ is a bounded domain with the smooth boundary $\partial \Omega$ such that $0 \in \Omega, \Delta u=\operatorname{div}(\nabla u)$ is the Laplacian operator, $1<q<2, \lambda, \mu>0, a_{i}, b_{i}>0(i=1,2)$, and $a, b, c \in C\left(\Omega, \mathbb{R}^{+}\right)$, the function $F \in C^{1}\left(\bar{\Omega} \times\left(\mathbb{R}^{+}\right)^{2}, \mathbb{R}^{+}\right)$, is positively homogeneous of degree $\alpha=4 \mathrm{~N} /(N-2)$ which is the Sobolev critical exponent; that is, $F(x, t u, t v)=$ $t^{\alpha} F(x, u, v),(t>0)$ holds for all $(x, u, v) \in \bar{\Omega} \times\left(\mathbb{R}^{+}\right)^{2}$, $\left(F_{u}, F_{v}\right)=\nabla F$.

In recent years, there have been many papers concerned with the existence of the positive solutions for Kirchhoff equation

$$
\begin{gathered}
-M\left(\int_{\Omega}|\nabla u|^{2} d x\right) \Delta u=\lambda f(x, u) \quad \text { in } \Omega \\
u=\Delta u=0 \quad \text { on } \partial \Omega,
\end{gathered}
$$

which is related to the stationary analogue of the Kirchhoff equation

$$
u_{t t}-M\left(\int_{\Omega}|\nabla u|^{2} d x\right) \Delta u=f(x, u)
$$

where $M(s)=a+b s, a, b>0$. It was proposed by Kirchhoff [1] as an extension of the classical D'Alembert wave equation for free vibrations of elastic strings.

Some interesting studies on these problems by variational methods can be found in [2-6]. As for perturbed fourthorder Kirchhoff-type elliptic problems, in [7] the following equation,

$$
\begin{gathered}
\Delta\left(|\Delta u|^{p-2} \Delta u\right)-\left[M\left(\int_{\Omega}|\nabla u|^{p} d x\right)\right]^{p-1} \Delta_{p} u+\rho|u|^{p-2} u \\
=\lambda f(x, u) \quad \text { in } \Omega \\
u=\Delta u=0 \quad \text { on } \partial \Omega,
\end{gathered}
$$

where $p>\max \{1, N / 2\}, \lambda>0, f: \Omega \times \mathbb{R} \rightarrow \mathbb{R}$ is an $L^{1}$-Carathéodory function, and $M:[0,+\infty[\rightarrow \mathbb{R}$ is a continuous function, has been investigated. The authors proved (4) has multiple nontrivial weak solutions. 
In [8] the authors established the existence of a weak solution for the following system equation:

$$
\begin{gathered}
-\left[M_{1}\left(\int_{\Omega}|\nabla u|^{p} d x\right)\right]^{p-1} \Delta_{p} u=f(u, v)+\rho_{1}(x) \quad \text { in } \Omega \\
-\left[M_{2}\left(\int_{\Omega}|\nabla v|^{p} d x\right)\right]^{p-1} \Delta_{p} v=f(u, v)+\rho_{2}(x) \text { in } \Omega \\
\frac{\partial u}{\partial \eta}=\frac{\partial v}{\partial \eta}=0 \quad \text { on } \partial \Omega,
\end{gathered}
$$

where $M_{1}(t), M_{2}(t) \geq m_{0}>0$.

Motivated by the results of the above cited papers, we will attempt to treat problem (1) and extend the results for our problem.

In this paper we make the following assumptions.

Let $S$ be the best Sobolev embedding constant defined by

$$
S=\inf _{u \in W_{0}^{1,2}(\Omega) \backslash\{0\}} \frac{\int_{\Omega}\left(|\nabla u|^{2}+a(x)|u|^{2}\right) d x}{\left(\int_{\Omega}|u|^{\alpha} d x\right)^{2 / \alpha}}
$$

and let $|\Omega|$ be the Lebesgue measure of $\Omega ;|\cdot|_{\infty}$ denotes the $L^{\infty}(\Omega) \operatorname{norm}, \beta=\min \left\{b_{1}, b_{2}\right\}$, and

$$
\begin{gathered}
C\left(q, N, K, S,|\Omega|, a_{1}, a_{2}\right) \\
=\frac{A(\alpha-2) S^{q / 2}}{(\alpha-q)|\Omega|^{(\alpha-q) / \alpha}}\left(\frac{A(2-q) S^{\alpha / 2}}{K(\alpha-q)}\right)^{(2-q) /(\alpha-2)}, \\
C_{0}=\left(\frac{q}{2}\right) C\left(q, N, K, S,|\Omega|, a_{1}, a_{2}\right),
\end{gathered}
$$

where $A=\min \left\{a_{1}, a_{2}\right\}$.

Also the following hold:

(F1) $F: \bar{\Omega} \times \mathbb{R}^{+} \times \mathbb{R}^{+} \rightarrow \mathbb{R}^{+}$is a $C^{1}$ function and $F(x, t u, t v)=t^{\alpha} F(x, u, v)$,

(F2) $F(x, u, 0)=F(x, 0, v)=F_{u}(x, u, 0)=F_{v}(x, 0, v)=0$ where $u, v \in \mathbb{R}^{+}$,

(F3) $F_{u}(x, u, v), F_{v}(x, u, v)$ are strictly increasing function about $u, v$ for all $u>0, v>0$.

In addition, using assumption (F1), we have the so-called Euler identity

$$
(u, v) \cdot \nabla F(x, u, v)=\alpha F(x, u, v)
$$

and, for a positive constant $K$,

$$
F(x, u, v) \leq K\left(|u|^{2}+|v|^{2}\right)^{\alpha / 2} .
$$

Let $W_{0}^{1,2}(\Omega)$ be the completion of $C_{0}^{\infty}(\Omega)$ with respect to the $\operatorname{norm}\left(\int_{\Omega}|\nabla u|^{2}\right)^{1 / 2}$.

It is easy to show that, for every $u \in W_{0}^{1,2}(\Omega)$, the above norm is equivalent with $\|u\|=\left(\int_{\Omega}|\nabla u|^{2}+a(x)|u|^{2}\right)^{1 / 2}$. Problem (1) is posed in the framework of the Sobolev space

$$
E=\left\{(u, v) \in\left(W_{0}^{1,2}(\Omega)\right)^{2} \mid \int_{\Omega} a(x)\left(|u|^{2}+|v|^{2}\right) d x<+\infty\right\},
$$

with the standard norm

$$
\begin{aligned}
& \|(u, v)\|_{E} \\
& \quad=\left(\int_{\Omega}\left(|\nabla u|^{2}+a(x)|u|^{2}\right) d x\right. \\
& \left.\quad+\int_{\Omega}\left(|\nabla v|^{2}+a(x)|v|^{2}\right) d x\right)^{1 / 2} .
\end{aligned}
$$

We will look for solutions of (1) by finding critical points of the energy functional $J_{\lambda, \mu}: E \rightarrow \mathbb{R}$ given by

$$
\begin{aligned}
J_{\lambda, \mu}(u, v)= & \frac{a_{1}}{2}\|u\|^{2}+\frac{a_{2}}{2}\|v\|^{2}+\frac{b_{1}}{4}\|u\|^{4} \\
& +\frac{b_{2}}{4}\|v\|^{4}-\frac{1}{\alpha} \int_{\Omega} F(x, u, v) d x-\frac{1}{q} K_{\lambda, \mu}(u, v),
\end{aligned}
$$

where $K_{\lambda, \mu}: E \rightarrow \mathbb{R}$ is the functional defined by

$$
K_{\lambda, \mu}=\int_{\Omega}\left(\lambda b(x)|u|^{q}+\mu c(x)|v|^{q}\right) d x .
$$

It is well known that the functional $J_{\lambda, \mu} \in C^{1}(E, \mathbb{R})$. For any $\left(\varphi_{1}, \varphi_{2}\right) \in E$, there holds

$$
\begin{aligned}
& \left\langle J_{\lambda, \mu}^{\prime}(u, v),(u, v)\right\rangle \\
& =\left(a_{1}+b_{1}\left(\int_{\Omega}\left(|\nabla u|^{2}+a(x)|u|^{2}\right) d x\right)\right) \\
& \quad \times\left(\int_{\Omega}\left(\nabla u \nabla \varphi_{1}+a(x) u \varphi_{1}\right) d x\right) \\
& \quad+\left(a_{2}+b_{2}\left(\int_{\Omega}\left(|\nabla v|^{2}+a(x)|v|^{2}\right) d x\right)\right) \\
& \quad \times\left(\int_{\Omega}\left(\nabla v \nabla \varphi_{2}+a(x) u \varphi_{2}\right) d x\right) \\
& \quad-\frac{1}{\alpha} \int_{\Omega}\left(F_{u}(x, u, v) \varphi_{1}+F_{v}(x, u, v) \varphi_{2}\right) d x \\
& \quad-\lambda \int_{\Omega} b(x)|u|^{q-2} u \varphi_{1} d x-\mu \int_{\Omega} c(x)|v|^{q-2} v \varphi_{2} d x .
\end{aligned}
$$

Consider the Nehari manifold

$$
N_{\lambda, \mu}=\left\{(u, v) \in E \backslash\{0,0\}\left\langle J_{\lambda, \mu}^{\prime}(u, v),(u, v)\right\rangle=0\right\} .
$$

Note that $(u, v) \in N_{\lambda, \mu}$ if and only if

$$
\begin{aligned}
& a_{1}\|u\|^{2}+a_{2}\|v\|^{2}+b_{1}\|u\|^{4}+b_{2}\|v\|^{4}-\int_{\Omega} F(x, u, v) d x \\
& -K_{\lambda, \mu}(u, v)=0 .
\end{aligned}
$$

So $N_{\lambda, \mu}$ contains all nontrivial weak solutions of (1). 

$N_{\lambda, \mu}$

Define $\Phi_{\lambda, \mu}(u, v)=\left\langle J_{\lambda, \mu}^{\prime}(u, v),(u, v)\right\rangle$. Then, for $(u, v) \in$

$$
\begin{aligned}
&\left\langle\Phi_{\lambda, \mu}^{\prime}(u, v),(u, v)\right\rangle \\
&=2 a_{1}\|u\|^{2}+2 a_{2}\|v\|^{2}+4 b_{1}\|u\|^{4} \\
& \quad+4 b_{2}\|v\|^{4}-\alpha \int_{\Omega} F(x, u, v) d x-q K_{\lambda, \mu}(u, v) \\
&=2 b_{1}\|u\|^{4}+2 b_{2}\|v\|^{4}-(\alpha-2) \int_{\Omega} F(x, u, v) d x \\
& \quad-(q-2) K_{\lambda, \mu}(u, v) \\
&=-2 a_{1}\|u\|^{2}-2 a_{2}\|v\|^{2}-(\alpha-4) \int_{\Omega} F(x, u, v) d x \\
& \quad-(q-4) K_{\lambda, \mu}(u, v) \\
&=(2-\alpha)\left(a_{1}\|u\|^{2}+a_{2}\|v\|^{2}\right) \\
&+(4-\alpha)\left(b_{1}\|u\|^{4}+b_{2}\|v\|^{4}\right)-(q-\alpha) K_{\lambda, \mu}(u, v) \\
&=(2-q)\left(a_{1}\|u\|^{2}+a_{2}\|v\|^{2}\right) \\
&+(4-q)\left(b_{1}\|u\|^{4}+b_{2}\|v\|^{4}\right)-(\alpha-q) \int_{\Omega} F(x, u, v) d x .
\end{aligned}
$$

Now, we split $N_{\lambda, \mu}$ into three parts:

$$
\begin{aligned}
& N_{\lambda, \mu}^{+}=\left\{(u, v) \in N_{\lambda, \mu} \mid\left\langle\Phi_{\lambda, \mu}^{\prime}(u, v),(u, v)\right\rangle>0\right\} ; \\
& N_{\lambda, \mu}^{0}=\left\{(u, v) \in N_{\lambda, \mu} \mid\left\langle\Phi_{\lambda, \mu}^{\prime}(u, v),(u, v)\right\rangle=0\right\} ; \\
& N_{\lambda, \mu}^{-}=\left\{(u, v) \in N_{\lambda, \mu} \mid\left\langle\Phi_{\lambda, \mu}^{\prime}(u, v),(u, v)\right\rangle<0\right\} .
\end{aligned}
$$

\section{Statement of the Main Results}

Let us first define $\varrho_{\lambda, \mu}=\left(\lambda|b|_{\infty}\right)^{2 /(2-q)}+\left(\mu|c|_{\infty}\right)^{2 /(2-q)}$ and the main results read as follows.

Theorem 1. If $(\lambda, \mu)$ satisfy $0<\varrho_{\lambda, \mu}<C\left(q, N, K, S,|\Omega|, a_{1}\right.$, $\left.a_{2}\right)$ and (F1)-(F3) hold, then problem (1) has at least one positive solution.

Theorem 2. If $(\lambda, \mu)$ satisfy $0<\varrho_{\lambda, \mu}<C_{0}^{*}$ and (F1)-(F3) hold, then problem (1) has at least two positive solutions.

Note that, using assumption (F3), we have that $F_{u}, F_{v} \in$ $C\left(\bar{\Omega} \times\left(\mathbb{R}^{+}\right)^{2}, \mathbb{R}^{+}\right)$are positively homogeneous of degree $\alpha-1$. This implies that

$$
\left|F_{u}(x, u, v)\right| \leq M\left(|u|^{\alpha-1}+|v|^{\alpha-1}\right),
$$

$\left|F_{v}(x, u, v)\right| \leq M\left(|u|^{\alpha-1}+|v|^{\alpha-1}\right), \quad \forall x \in \bar{\Omega}, u, v \in \mathbb{R}^{+}$, for some positive constant $M$. Similar to Willem [9, Theorem A.2], we consider the continuity of the superposition operator

$$
A: L^{2}(\Omega) \longrightarrow L^{q}(\Omega):(u, v) \longmapsto f(x, u, v) .
$$

Lemma 3. Assume that $|\Omega|<\infty, r<\infty, f \in C\left(\bar{\Omega} \times \mathbb{R}^{2}, \mathbb{R}\right)$, and

$$
|f(x, u, v)| \leq c\left(1+|u|^{2 / r}+|v|^{2 / r}\right) .
$$

Then, for every $(u, v) \in L^{2}(\Omega), f(\cdot, u, v) \in L^{r}(\Omega)$ and the operator $A: L^{p}(\Omega) \rightarrow L^{r}(\Omega):(u, v) \mapsto f(x, u, v)$ is continuous.

Now, we consider the functional $\psi(u, v)=\int_{\Omega} F(x, u, v) d x$; then we have the following result.

Lemma 4. Assume that $|\Omega|<\infty, F \in C\left(\bar{\Omega} \times\left(\mathbb{R}^{+}\right)^{2}, \mathbb{R}^{+}\right)$ satisfying (F3); then the functional $\psi$ is of class $C^{1}\left(E, \mathbb{R}^{+}\right)$and

$$
\left\langle\psi^{\prime}(u, v),\left(\varphi_{1}, \varphi_{2}\right)\right\rangle=\int_{\Omega}\left(F_{u}(x, u, v) \varphi_{1}+F_{v}(x, u, v) \varphi_{2}\right) d x
$$

where $(u, v),\left(\varphi_{1}, \varphi_{2}\right) \in E$.

Proof. The proof is almost the same as that in [10].

Lemma 5. The energy functional $J_{\lambda, \mu}$ is coercive and bounded below on $N_{\lambda, \mu}$.

Proof. If $(u, v) \in N_{\lambda, \mu}$, then by the Hölder inequality and the Sobolev embedding theorem

$$
\begin{aligned}
J_{\lambda, \mu}(u, v)= & \frac{\alpha-2}{2 \alpha}\left(a_{1}\|u\|^{2}+a_{2}\|v\|^{2}\right) \\
& +\frac{\alpha-4}{4 \alpha}\left(b_{1}\|u\|^{4}+b_{2}\|v\|^{4}\right)-\frac{\alpha-q}{\alpha q} K_{\lambda, \mu}(u, v) \\
\geq & \frac{\alpha-2}{2 \alpha}\left(a_{1}\|u\|^{2}+a_{2}\|v\|^{2}\right)+\frac{\alpha-4}{4 \alpha}\left(b_{1}\|u\|^{4}+b_{2}\|v\|^{4}\right) \\
& -\frac{\alpha-q}{\alpha q} \varrho_{\lambda, \mu}^{(2-q) / 2} S^{-q / 2}|\Omega|^{(\alpha-q) / \alpha}\|(u, v)\|_{E}^{q} .
\end{aligned}
$$

Thus, $J_{\lambda, \mu}$ is coercive and bounded below on $N_{\lambda, \mu}$.

Lemma 6. Suppose that $\left(u_{0}, v_{0}\right)$ is a local minimizer for $J_{\lambda, \mu}$ on $N_{\lambda, \mu}$ and that $\left(u_{0}, v_{0}\right) \notin N_{\lambda, \mu}^{0}$. Then $J_{\lambda, \mu}^{\prime}\left(u_{0}, v_{0}\right)=0$ in $E^{-1}$ (the dual space of the Sobolev space E).

Proof. If $\left(u_{0}, v_{0}\right)$ is a local minimizer for $J_{\lambda, \mu}$ on $N_{\lambda, \mu}$, then $\left(u_{0}, v_{0}\right)$ is a solution of the optimization problem minimizer $J_{\lambda, \mu}(u, v)$ subject to $\Phi_{\lambda, \mu}(u, v)=0$. Hence, by the theory of Lagrange multipliers, there exists $\xi_{1} \in R$, such that

$$
J_{\lambda, \mu}^{\prime}\left(u_{0}, v_{0}\right)=\xi_{1} \Phi_{\lambda, \mu}^{\prime}\left(u_{0}, v_{0}\right) \quad \text { in } E^{-1}(\Omega),
$$

and thus,

$$
\left\langle J_{\lambda, \mu}^{\prime}\left(u_{0}, v_{0}\right),\left(u_{0}, v_{0}\right)\right\rangle=\xi_{1}\left\langle\Phi_{\lambda, \mu}^{\prime}\left(u_{0}, v_{0}\right),\left(u_{0}, v_{0}\right)\right\rangle .
$$


Since $\left(u_{0}, v_{0}\right) \in N_{\lambda, \mu}$, we have $\left\langle J_{\lambda, \mu}^{\prime}\left(u_{0}, v_{0}\right),\left(u_{0}, v_{0}\right)\right\rangle=0$. Moreover, $\left\langle\Phi_{\lambda, \mu}^{\prime}\left(u_{0}, v_{0}\right),\left(u_{0}, v_{0}\right)\right\rangle \neq 0$, so $\xi_{1}=0$. This completes the proof.

Lemma 7. If $0<\varrho_{\lambda, \mu}<C\left(q, N, K, S,|\Omega|, a_{1}, a_{2}\right)$, then $N_{\lambda, \mu}^{0}=$ $\emptyset$.

Proof. Suppose otherwise that $0<\varrho_{\lambda, \mu}<C(q, N, K, S,|\Omega|$, $\left.a_{1}, a_{2}\right)$ such that $N_{\lambda, \mu}^{0} \neq \emptyset$.

Then, for $(u, v) \in N_{\lambda, \mu}^{0}$,

$$
\begin{aligned}
0= & \left\langle\Phi_{\lambda, \mu}^{\prime}(u, v),(u, v)\right\rangle \\
= & (2-\alpha)\left(a_{1}\|u\|^{2}+a_{2}\|v\|^{2}\right)+(4-\alpha)\left(b_{1}\|u\|^{4}+b_{2}\|v\|^{4}\right) \\
& -(q-\alpha) K_{\lambda, \mu}(u, v) \\
= & (2-q)\left(a_{1}\|u\|^{2}+a_{2}\|v\|^{2}\right)+\left(b_{1}\|u\|^{4}+b_{2}\|v\|^{4}\right) \\
& -(\alpha-q) \int_{\Omega} F(x, u, v) d x .
\end{aligned}
$$

By the Holder inequality and the Sobolev embedding theorem

$$
\begin{aligned}
& (\alpha-2)\left(a_{1}\|u\|^{2}+a_{2}\|v\|^{2}\right)+(\alpha-4)\left(b_{1}\|u\|^{4}+b_{2}\|v\|^{4}\right) \\
& \quad=(\alpha-q) K_{\lambda, \mu}(u, v) \\
& \quad \leq(\alpha-q) \varrho_{\lambda, \mu}^{(2-q) / 2} S^{-q / 2}|\Omega|^{(\alpha-q) / \alpha}\|(u, v)\|_{E}^{q} .
\end{aligned}
$$

So,

$$
\begin{aligned}
A(\alpha-2)\|(u, v)\|_{E}^{2} & \leq(\alpha-2)\left(a_{1}\|u\|^{2}+a_{2}\|v\|^{2}\right) \\
& \leq(q-\alpha) \varrho_{\lambda, \mu}^{(2-q) / 2} S^{-q / 2}|\Omega|^{(\alpha-q) / \alpha}\|(u, v)\|_{E}^{q} .
\end{aligned}
$$

Thus,

$$
\|(u, v)\|_{E} \leq\left(\frac{(\alpha-q) \varrho_{\lambda, \mu}^{(2-q) / 2} S^{-q / 2}|\Omega|^{(\alpha-q) / \alpha}}{A(\alpha-2)}\right)^{1 /(2-q)}
$$

and, by the Minkowski inequality, the Sobolev embedding theorem, and (9),

$$
\begin{aligned}
\int_{\Omega} F & (x, u, v) d x \leq K\left(\int_{\Omega}\left(|u|^{2}+|v|^{2}\right)^{\alpha / 2} d x\right)^{(2 / \alpha) \cdot(\alpha / 2)} \\
& \leq K\left(\left(\int_{\Omega}|u|^{\alpha} d x\right)^{2 / \alpha}+\left(\int_{\Omega}|v|^{\alpha} d x\right)^{2 / \alpha}\right)^{\alpha / 2} \\
& \leq K S^{-\alpha / 2}\|(u, v)\|_{E}^{\alpha} .
\end{aligned}
$$

Thus,

$$
\|(u, v)\|_{E} \geq\left(\frac{A(2-q) S^{\alpha / 2}}{K(\alpha-q)}\right)^{1 /(\alpha-2)}
$$

This implies that

$$
\varrho_{\lambda, \mu} \geq C\left(q, N, K, S,|\Omega|, a_{1}, a_{2}\right)
$$

which is a contradiction. Thus, we can conclude that if

$$
0<\varrho_{\lambda, \mu}<C\left(q, N, K, S,|\Omega|, a_{1}, a_{2}\right),
$$

we have $N_{\lambda, \mu}^{0}=\emptyset$.

By Lemma 7, we write $N_{\lambda, \mu}=N_{\lambda, \mu}^{+} \cup N_{\lambda, \mu}^{-}$and define

$$
\begin{aligned}
& \theta_{\lambda, \mu}=\inf _{(u, v) \in N_{\lambda, \mu}} J_{\lambda, \mu}(u, v) ; \\
& \theta_{\lambda, \mu}^{+}=\inf _{(u, v) \in N_{\lambda, \mu}^{+}} J_{\lambda, \mu}(u, v) ; \\
& \theta_{\lambda, \mu}^{-}=\inf _{(u, v) \in N_{\lambda, \mu}^{-}} J_{\lambda, \mu}(u, v) .
\end{aligned}
$$

Then we have the following result.

Lemma 8. Consider the following.

(i) If $0<\varrho_{\lambda, \mu}<C\left(q, N, K, S,|\Omega|, a_{1}, a_{2}\right)$, then one has $\theta_{\lambda, \mu} \leq \theta_{\lambda, \mu}^{+}<0$.

(ii) If $0<\varrho_{\lambda, \mu}<C_{0}$, then $\theta_{\lambda, \mu}^{-}>d_{0}$ for some constant

$$
d_{0}=d_{0}\left(q, N, K, S,|\Omega|, \varrho_{\lambda, \mu}, a_{1}, a_{2}, b_{1}, b_{2}\right)>0 .
$$

Proof. (i) Let $(u, v) \in N_{\lambda, \mu}^{+}$. Then

$$
\begin{aligned}
& (2-q)\left(a_{1}\|u\|^{2}+a_{2}\|v\|^{2}\right)+(4-q)\left(b_{1}\|u\|^{4}+b_{2}\|v\|^{4}\right) \\
& -(\alpha-q) \int_{\Omega} F(x, u, v) d x>0
\end{aligned}
$$

and so

$$
\begin{aligned}
J_{\lambda, \mu}(u, v)= & \left(\frac{1}{2}-\frac{1}{q}\right)\left(a_{1}\|u\|^{2}+a_{2}\|v\|^{2}\right) \\
& +\left(\frac{1}{4}-\frac{1}{q}\right)\left(b_{1}\|u\|^{4}+b_{2}\|v\|^{4}\right) \\
& +\left(\frac{1}{q}-\frac{1}{\alpha}\right) \int_{\Omega} F(x, u, v) d x \\
< & \frac{q-2}{q}\left(\frac{1}{2}-\frac{1}{\alpha}\right)\left(a_{1}\|u\|^{2}+a_{2}\|v\|^{2}\right) \\
& +\frac{q-4}{q}\left(\frac{1}{4}-\frac{1}{\alpha}\right)\left(b_{1}\|u\|^{4}+b_{2}\|v\|^{4}\right)<0 .
\end{aligned}
$$

Thus, from the definition of $\theta_{\lambda, \mu}$ and $\theta_{\lambda, \mu}^{+}$, we can deduce that $\theta_{\lambda, \mu} \leq \theta_{\lambda, \mu}^{+}<0$. 
(ii) Let $(u, v) \in N_{\lambda, \mu}^{-}$. Then

$$
\begin{aligned}
& (2-q)\left(a_{1}\|u\|^{2}+a_{2}\|v\|^{2}\right)+(4-q)\left(b_{1}\|u\|^{4}+b_{2}\|v\|^{4}\right) \\
& -(\alpha-q) \int_{\Omega} F(x, u, v) d x<0 .
\end{aligned}
$$

By (8) we have

$$
\begin{aligned}
& (2-q)\left(a_{1}\|u\|^{2}+a_{2}\|v\|^{2}\right)+(4-q)\left(b_{1}\|u\|^{4}+b_{2}\|v\|^{4}\right) \\
& \quad<(\alpha-q) \int_{\Omega} F(x, u, v) d x \\
& \quad \leq(\alpha-q) K S^{-\alpha / q}\|(u, v)\|_{E}^{\alpha} .
\end{aligned}
$$

This implies that

$$
\|(u, v)\|_{E}>\left(\frac{A(2-q) S^{\alpha / q}}{K(\alpha-q)}\right)^{1 /(\alpha-2)} .
$$

By (23) in the proof of Lemma 5,

$$
\begin{aligned}
& J_{\lambda, \mu}(u, v) \\
& \geq A\left(\frac{1}{2}-\frac{1}{\alpha}\right)\|(u, v)\|_{E}^{2} \\
&-\left(\frac{1}{q}-\frac{1}{\alpha}\right) \varrho_{\lambda, \mu}^{(2-q) / 2} S^{-q / 2}|\Omega|^{(\alpha-q) / \alpha}\|(u, v)\|_{E}^{q} \\
&> A\left(\frac{1}{2}-\frac{1}{\alpha}\right)\left(\frac{A(q-2) S^{\alpha / q}}{K(\alpha-q)}\right)^{2 /(\alpha-2)} \\
&-\left(\frac{1}{q}-\frac{1}{\alpha}\right) \varrho_{\lambda, \mu}^{(2-q) / 2} S^{(N-q) / 2}|\Omega|^{(\alpha-q) / \alpha} \\
& \times\left(\frac{A(q-2) S^{\alpha / q}}{K(\alpha-q)}\right)^{q /(\alpha-2)} .
\end{aligned}
$$

Thus, if $0<\lambda<C_{0}$, then

$$
J_{\lambda, \mu}(u, v)>d_{0} \quad \forall(u, v) \in N_{\lambda, \mu}^{-},
$$

for some $d_{0}=d_{0}\left(\lambda, \mu, q, N, K, S,|\Omega|,|b|_{\infty},|c|_{\infty}, a_{1}, a_{2}, b_{1}\right.$, $\left.b_{2}\right)>0$. This completes the proof.

For each $(u, v) \in E$ with $\int_{\Omega} F(x, u, v) d x>0$, set

$$
\begin{aligned}
t_{\max }=( & \left((2-q)\left(a_{1}\|u\|^{2}+a_{2}\|v\|^{2}\right)\right. \\
& \left.+(4-q)\left(b_{1}\|u\|^{4}+b_{2}\|v\|^{4}\right)\right) \\
& \left.\times\left((\alpha-q) \int_{\Omega} F(x, u, v) d x\right)^{-1}\right)^{1 /(\alpha-2)}
\end{aligned}
$$

$>0$.

Then we have the following.
Lemma 9 (see [11, Lemma 2.6]). For each $(u, v) \in E$ with $\int_{\Omega} F(x, u, v) d x>0$, there are unique $0<t^{+}<t_{\max }<t^{-}$such that $\left(t^{+} u, t^{+} v\right) \in N_{\lambda, \mu}^{+},\left(t^{-} u, t^{-} v\right) \in N_{\lambda, \mu}^{-}$and $J_{\lambda, \mu}\left(t^{+} u, t^{+} v\right)=$ $\inf _{0 \leq t \leq t_{\max }} J_{\lambda, \mu}(t u, t v) ; J_{\lambda, \mu}\left(t^{-} u, t^{-} v\right)=\sup _{t \geq 0} J_{\lambda, \mu}(t u, t v)$.

\section{Proof of the Main Theorems}

We will need the following lemma.

Lemma 10 (see [12]). Consider the following.

(i) If $0<\varrho_{\lambda, \mu}^{(2-q) / 2}<C\left(q, N, K, S,|\Omega|, \varrho_{\lambda, \mu}, a_{1}, a_{2}\right)$, then there exists a $(P S)_{\theta_{\lambda, \mu}}$-sequence $\left\{\left(u_{n}, v_{n}\right)\right\} \subset N_{\lambda, \mu}$ in $E$ for $J_{\lambda, \mu}$.

(ii) If $0<\varrho_{\lambda, \mu}<C_{0}$, then there exists a $(P S)_{\theta_{\lambda, \mu}^{-}}$-sequence $\left\{\left(u_{n}, v_{n}\right)\right\} \subset N_{\lambda, \mu}^{-}$in $E$ for $J_{\lambda, \mu}$.

Theorem 11. If $0<\varrho_{\lambda, \mu}<C\left(q, N, K, S,|\Omega|, \varrho_{\lambda, \mu}, a_{1}, a_{2}\right)$ and (F1)-(F3) hold, then $J_{\lambda, \mu}$ has a minimizer $\left(u_{0}^{+}, v_{0}^{+}\right)$in $N_{\lambda, \mu}^{+}$and it satisfies the following:

(i) $J_{\lambda, \mu}\left(u_{0}^{+}, v_{0}^{+}\right)=\theta_{\lambda, \mu}=\theta_{\lambda, \mu}^{+}$;

(ii) $\left(u_{0}^{+}, v_{0}^{+}\right)$is a positive solution of (1).

Proof. By Lemma 10(i), there exists a minimizing sequence $\left\{\left(u_{n}, v_{n}\right)\right\}$ for $J_{\lambda, \mu}$ on $N_{\lambda, \mu}$ such that

$$
J_{\lambda, \mu}\left(u_{n}, v_{n}\right)=\theta_{\lambda, \mu}+o(1), \quad J_{\lambda, \mu}^{\prime}\left(u_{n}, v_{n}\right)=o(1) .
$$

Then by Lemma 5 and the compact imbedding theorem, there exist a subsequence $\left\{\left(u_{n}, v_{n}\right)\right\}$ and $\left(u_{0}^{+}, v_{0}^{+}\right) \in E$ such that

$$
\begin{gathered}
u_{n} \rightarrow u_{0}^{+} \quad \text { weakly in } W_{0}^{1,2}, \\
u_{n} \longrightarrow u_{0}^{+} \quad \text { strongly in } L^{q}(\Omega), \\
v_{n} \rightarrow v_{0}^{+} \quad \text { weakly in } W_{0}^{1,2}, \\
v_{n} \longrightarrow v_{0}^{+} \quad \text { strongly in } L^{q}(\Omega) .
\end{gathered}
$$

This implies that $K_{\lambda, \mu}\left(u_{n}, v_{n}\right) \rightarrow K_{\lambda, \mu}\left(u_{0}^{+}, v_{0}^{+}\right)$as $n \rightarrow \infty$. By (44) and (45), it is easy to prove that $\left(u_{0}^{+}, v_{0}^{+}\right)$is $s$ weak solution of (1). Since

$$
\begin{aligned}
J_{\lambda, \mu}\left(u_{n}, v_{n}\right)= & \frac{(N+2)}{4 N}\left(a_{1}\|u\|^{2}+a_{2}\|v\|^{2}\right) \\
& +\frac{1}{2 N}\left(b_{1}\|u\|^{4}+b_{2}\|v\|^{4}\right) \\
& -\frac{\alpha-q}{\alpha q} K_{\lambda, \mu}\left(u_{n}, v_{n}\right) \\
\geq & -\frac{\alpha-q}{\alpha q} K_{\lambda, \mu}\left(u_{n}, v_{n}\right)
\end{aligned}
$$

and by Lemma $8(\mathrm{i})$,

$$
J_{\lambda, \mu}\left(u_{n}, v_{n}\right) \longrightarrow \theta_{\lambda, \mu}<0 \quad \text { as } n \longrightarrow \infty \text {. }
$$


Letting $n \rightarrow \infty$, we see that $K_{\lambda, \mu}\left(u_{0}^{+}, v_{0}^{+}\right)>0$. Thus, $\left(u_{0}^{+}, v_{0}^{+}\right)$ is a nontrivial solution of problem (1). Now it follows that $u_{n} \rightarrow u_{0}^{+}$strongly in $W_{0}^{1,2}$ and $v_{n} \rightarrow v_{0}^{+}$strongly in $W_{0}^{1,2}$ and $J_{\lambda, \mu}\left(u_{0}^{+}, v_{0}^{+}\right)=\theta_{\lambda, \mu}$. By $\left(u_{0}^{+}, v_{0}^{+}\right) \in N_{\lambda, \mu}$ and applying Fatou's lemma, we get

$$
\begin{aligned}
\theta_{\lambda, \mu} \leq J_{\lambda, \mu}\left(u_{0}^{+}, v_{0}^{+}\right)= & \frac{N+2}{4 N}\left(a_{1}\left\|u_{0}^{+}\right\|^{2}+a_{2}\left\|v_{0}^{+}\right\|^{2}\right) \\
& \times \frac{1}{2 N}\left(b_{1}\left\|u_{0}^{+}\right\|^{4}+b_{2}\left\|v_{0}^{+}\right\|^{4}\right) \\
& -\frac{\alpha-q}{\alpha q} K_{\lambda, \mu}\left(u_{0}^{+}, v_{0}^{+}\right) \\
\leq & \liminf _{n \rightarrow \infty}\left(\frac{N+2}{4 N}\left(a_{1}\left\|u_{n}\right\|^{2}+a_{2}\left\|v_{n}\right\|^{2}\right)\right. \\
& +\frac{1}{2 N}\left(b_{1}\|u\|^{4}+b_{2}\|v\|^{4}\right) \\
& \left.-\frac{\alpha-q}{\alpha q} K_{\lambda, \mu}\left(u_{n}, v_{n}\right)\right) \\
\leq & \liminf _{n \rightarrow \infty} J_{\lambda, \mu}\left(u_{n}, v_{n}\right)=\theta_{\lambda, \mu} .
\end{aligned}
$$

This implies that

$$
\begin{gathered}
J_{\lambda, \mu}\left(u_{0}^{+}, v_{0}^{+}\right)=\theta_{\lambda, \mu} ; \quad \lim _{n \rightarrow \infty}\left\|u_{n}\right\|^{2}=\left\|u_{0}^{+}\right\|^{2}, \\
\lim _{n \rightarrow \infty}\left\|v_{n}\right\|^{2}=\left\|v_{0}^{+}\right\|^{2} .
\end{gathered}
$$

Let $\tilde{u}_{n}=u_{n}-u_{0}^{+}, \widetilde{v}_{n}=v_{n}-v_{0}^{+}$; then by the Brezis-Lieb lemma [13], this implies

$$
\begin{gathered}
\left\|\widetilde{u}_{n}\right\|^{2}=\left\|u_{n}\right\|^{2}-\left\|u_{0}^{+}\right\|^{2}, \\
\left\|\widetilde{v}_{n}\right\|^{2}=\left\|v_{n}\right\|^{2}-\left\|v_{0}^{+}\right\|^{2} .
\end{gathered}
$$

Therefore, $u_{n} \rightarrow u_{0}^{+}$strongly in $W_{0}^{1,2}$ and $v_{n} \rightarrow v_{0}^{+}$ strongly in $W_{0}^{1,2}$. Moreover, we have $\left(u_{0}^{+}, v_{0}^{+}\right) \in N_{\lambda, \mu}^{+}$. In fact, if $\left(u_{0}^{+}, v_{0}^{+}\right) \in N_{\lambda, \mu}^{-}$, by Lemma 9 , there are unique $t_{0}^{+}$and $t_{0}^{-}$ such that $\left(t_{0}^{+} u_{0}^{+}, t_{0}^{+} v_{0}^{+}\right) \in N_{\lambda, \mu}^{+}$and $\left(t_{0}^{-} u_{0}^{+}, t_{0}^{-} v_{0}^{+}\right) \in N_{\lambda, \mu}^{-}$. In particular, we have $t_{0}^{+}<t_{0}^{-}=1$. Since

$$
\frac{d}{d t} J_{\lambda, \mu}\left(t_{0}^{+} u_{0}^{+}, t_{0}^{+} v_{0}^{+}\right)=0, \quad \frac{d^{2}}{d t^{2}} J_{\lambda, \mu}\left(t_{0}^{+} u_{0}^{+}, t_{0}^{+} v_{0}^{+}\right)>0,
$$

there exists $t_{0}^{+}<\bar{t} \leq t_{0}^{-}$such that $J_{\lambda, \mu}\left(t_{0}^{+} u_{0}^{+}, t_{0}^{+} v_{0}^{+}\right)<J_{\lambda, \mu}\left(\bar{t} u_{0}^{+}\right.$, $\left.\bar{t} v_{0}^{+}\right)$. By Lemma 9,

$$
\begin{aligned}
J_{\lambda, \mu}\left(t_{0}^{+} u_{0}^{+}, t_{0}^{+} v_{0}^{+}\right) & <J_{\lambda, \mu}\left(\bar{t} u_{0}^{+}, \bar{t} v_{0}^{+}\right) \leq J_{\lambda, \mu}\left(t_{0}^{-} u_{0}^{+}, t_{0}^{-} v_{0}^{+}\right) \\
& =J_{\lambda, \mu}\left(u_{0}^{+}, v_{0}^{+}\right)
\end{aligned}
$$

which is a contradiction. It follows from the maximum principle that $\left(u_{0}^{+}, v_{0}^{+}\right)$is a positive solution of problem (1). This completes the proof.
The following two lemmas are similar to those in [14].

Lemma 12. If $\left\{\left(u_{n}, v_{n}\right)\right\} \subset E$ is a $(P S)_{c}$-sequence for $J_{\lambda, \mu}$ with $\left(u_{n}, v_{n}\right) \rightarrow(u, v)$ in $E$, then $J_{\lambda, u}^{\prime}(u, v)=0$, and there exists $a$ positive constant $\Lambda$, such that $J_{\lambda, \mu}(u, v) \geq-\Lambda \varrho_{\lambda, \mu}$.

Lemma 13. If $\left\{\left(u_{n}, v_{n}\right)\right\} \subset E$ is a $(P S)_{c}$-sequence for $J_{\lambda, \mu}$, then $\left\{\left(u_{n}, v_{n}\right)\right\}$ is bounded in $E$.

Define

$$
\begin{gathered}
S_{F}:=\inf _{(u, v) \in E}\left\{\frac{a_{1}\|u\|^{2}+a_{2}\|v\|^{2}+b_{1}\|u\|^{4}+b_{2}\|v\|^{4}}{\left(\int_{\Omega} F(x, u, v) d x\right)^{2 / \alpha}}:\right. \\
\left.\int_{\Omega} F(x, u, v) d x>0\right\} .
\end{gathered}
$$

In addition, we need the following version of the Brezis-Lieb lemma [13].

Lemma 14. Consider $F \in C^{1}\left(\bar{\Omega},\left(\mathbb{R}^{+}\right)^{2}\right)$ with $F(x, 0,0)=0$ and

$$
\left|\frac{\partial F(x, u, v)}{\partial u}\right|,\left|\frac{\partial F(x, u, v)}{\partial v}\right| \leq C_{1}(|u|+|v|)
$$

for some $C_{1}>0$. Let $\left\{\left(u_{k}, v_{k}\right)\right\}$ be a bounded sequence in $L^{2}\left(\bar{\Omega},\left(\mathbb{R}^{+}\right)^{2}\right)$ such that $\left(u_{k}, v_{k}\right) \rightarrow(u, v)$ weakly in $E$. Then as $k \rightarrow \infty$,

$$
\begin{aligned}
\int_{\Omega} F\left(x, u_{k}, v_{k}\right) d x \rightarrow & \int_{\Omega} F\left(x, u_{k}-u, v_{k}-v\right) d x \\
& +\int_{\Omega} F(x, u, v) d x
\end{aligned}
$$

Lemma 15. $J_{\lambda, \mu}$ satisfies the $(P S)_{c}$ condition with $c$ satisfying

$$
-\infty<c<c_{\infty}=\left(\frac{1}{4}-\frac{1}{\alpha}\right) S_{F}^{2 N /(N+2)}-\Lambda \varrho_{\lambda, \mu} .
$$

Proof. Let $\left\{\left(u_{n}, v_{n}\right)\right\} \subset E$ be a $(P S)_{c}$-sequence for $J_{\lambda, \mu}$ with $c \in$ $\left(-\infty, c_{\infty}\right)$. It follows from Lemma 13 that $\left\{\left(u_{n}, v_{n}\right)\right\}$ is bounded in $E$, and then $\left(u_{n}, v_{n}\right) \rightarrow(u, v)$ up to a subsequence, where $(u, v)$ is a critical point of $J_{\lambda, \mu}$. Furthermore, we may assume

$$
\begin{gathered}
u_{n} \rightarrow u, \quad v_{n} \rightarrow v \quad \text { in } W_{0}^{1,2} \\
u_{n} \longrightarrow u, \quad v_{n} \longrightarrow v \quad \text { in } L^{q}(\Omega) \\
u_{n} \longrightarrow u, \quad v_{n} \longrightarrow v \quad \text { a.e. on } \Omega .
\end{gathered}
$$

Hence we have that $J_{\lambda, \mu}^{\prime}(u, v)=0$ and

$$
K_{\lambda, \mu}\left(u_{n}, v_{n}\right) \longrightarrow K_{\lambda, \mu}(u, v) \text {. }
$$

Let $\widetilde{u}_{n}=u_{n}-u, \widetilde{v}_{n}=v_{n}-v$. Then by the Brezis-Lieb lemma [13], we obtain

$$
\left\|\widetilde{u}_{n}\right\|^{2} \longrightarrow\left\|u_{n}\right\|^{2}-\|u\|^{2}, \quad\left\|\widetilde{v}_{n}\right\|^{2} \longrightarrow\left\|v_{n}\right\|^{2}-\|v\|^{2} \text { as } n \longrightarrow \infty,
$$


and by Lemma 14,

$$
\int_{\Omega} F\left(x, \widetilde{u}_{n}, \widetilde{v}_{n}\right) d x \longrightarrow \int_{\Omega} F\left(x, u_{n}, v_{n}\right) d x-\int_{\Omega} F(x, u, v) d x .
$$

Since $J_{\lambda, \mu}\left(u_{n}, v_{n}\right)=c+o(1), J_{\lambda, \mu}^{\prime}\left(u_{n}, v_{n}\right)=o(1)$ and (58)-(60), we can deduce that

$$
\begin{aligned}
\frac{a_{1}}{2}\left\|\tilde{u}_{n}\right\|^{2}+\frac{a_{2}}{2}\left\|\widetilde{v}_{n}\right\|^{2}+\frac{b_{1}}{4}\left\|\tilde{u}_{n}\right\|^{4}+\frac{b_{2}}{4}\left\|\widetilde{v}_{n}\right\|^{4} \\
\quad-\frac{1}{\alpha} \int_{\Omega} F\left(x, \widetilde{u}_{n}, \widetilde{v}_{n}\right) d x \\
=c-J_{\lambda, \mu}(u, v)+o(1) .
\end{aligned}
$$

So

$$
\begin{gathered}
\frac{1}{4}\left(a_{1}\left\|\widetilde{u}_{n}\right\|^{2}+a_{2}\left\|\widetilde{v}_{n}\right\|^{2}+b_{1}\left\|\tilde{u}_{n}\right\|^{4}+b_{2}\left\|\widetilde{v}_{n}\right\|^{4}\right) \\
\quad-\frac{1}{\alpha} \int_{\Omega} F\left(x, \widetilde{u}_{n}, \widetilde{v}_{n}\right) d x \\
\leq \frac{a_{1}}{2}\left\|\widetilde{u}_{n}\right\|^{2}+\frac{a_{2}}{2}\left\|\widetilde{v}_{n}\right\|^{2}+\frac{b_{1}}{4}\left\|\widetilde{u}_{n}\right\|^{4} \\
+\frac{b_{2}}{4}\left\|\widetilde{v}_{n}\right\|^{4}-\frac{1}{\alpha} \int_{\Omega} F\left(x, \widetilde{u}_{n}, \widetilde{v}_{n}\right) d x \\
=c-J_{\lambda, \mu}(u, v)+o(1), \\
a_{1}\left\|\widetilde{u}_{n}\right\|^{2}+a_{2}\left\|\widetilde{v}_{n}\right\|^{2}+b_{1}\left\|\widetilde{u}_{n}\right\|^{4}+b_{2}\left\|\widetilde{v}_{n}\right\|^{4} \\
-\int_{\Omega} F\left(x, \widetilde{u}_{n}, \widetilde{v}_{n}\right) d x=o(1) .
\end{gathered}
$$

Hence, we may assume that

$$
\begin{gathered}
a_{1}\left\|\tilde{u}_{n}\right\|^{2}+a_{2}\left\|\widetilde{v}_{n}\right\|^{2}+b_{1}\left\|\widetilde{u}_{n}\right\|^{4}+b_{2}\left\|\widetilde{v}_{n}\right\|^{4} \longrightarrow l, \\
\int_{\Omega} F\left(x, \widetilde{u}_{n}, \widetilde{v}_{n}\right) d x \longrightarrow l .
\end{gathered}
$$

If $l=0$, the proof is complete. Assume $l>0$; then from (63), we obtain

$$
\begin{aligned}
S_{F} l^{2 / \alpha} & =S_{F_{n}} \lim _{n \rightarrow \infty}\left(\int_{\Omega} F\left(x, \tilde{u}_{n}, \widetilde{v}_{n}\right) d x\right)^{2 / \alpha} \\
& \leq \lim _{n \rightarrow \infty}\left(a_{1}\left\|\widetilde{u}_{n}\right\|^{2}+a_{2}\left\|\widetilde{v}_{n}\right\|^{2}+b_{1}\left\|\widetilde{u}_{n}\right\|^{4}+b_{2}\left\|\tilde{v}_{n}\right\|^{4}\right)=l
\end{aligned}
$$

which implies that $l \geq S_{F}^{2 N /(N+2)}$. In addition, from Lemma 12, (61), and (63), we get

$$
c \geq\left(\frac{1}{4}-\frac{1}{\alpha}\right) l+J_{\lambda, \mu}(u, v) \geq\left(\frac{1}{4}-\frac{1}{\alpha}\right) S_{F}^{2 N /(N+2)}-\Lambda \varrho_{\lambda, \mu},
$$

which contradicts $c<(1 / 4-1 / \alpha) S_{F}^{2 N /(N+2)}-\Lambda \varrho_{\lambda, \mu}$.
Lemma 16. There exists a nonnegative function $(u, v) \in E \backslash$ $\{(0,0)\}$ and $C^{*}>0$ such that, for $\varrho_{\lambda, \mu} \in\left(0, C^{*}\right)$, one has

$$
\sup _{t \geq 0} J_{\lambda, \mu}(t u, t v)<c_{\infty} .
$$

In particular, $\theta_{\lambda, \mu}^{-}<c_{\infty}$ for all $\varrho_{\lambda, \mu} \in\left(0, C^{*}\right)$.

Proof. Since $0 \in \Omega$, there is $\rho_{0}>0$ such that $B^{N}\left(0 ; 2 \rho_{0}\right) \subset \Omega$. Now, we consider the functional $I: E \rightarrow R$ defined by

$I(u, v)=\frac{a_{1}}{2}\|u\|^{2}+\frac{a_{2}}{2}\|v\|^{2}-\frac{1}{\alpha} \int_{\Omega} F(x, u, v) d x \quad \forall(u, v) \in E$

and define a cut-off function $\eta(x) \in C_{0}^{\infty}(\Omega)$ such that $\eta(x)=$ 1 for $|x|<\rho_{0}, \eta(x)=0$ for $|x|>2 \rho_{0}, 0 \leq \eta \leq 1$, and $|\nabla \eta| \leq C$. For $\varepsilon>0$, let

$$
u_{\varepsilon}(x)=\frac{\eta(x)}{\left(\varepsilon+|x|^{2}\right)^{(N-2) / 2}} .
$$

From [14], we have the following estimates:

$$
\begin{gathered}
\left(\int_{\Omega}\left|u_{\varepsilon}\right|^{\alpha} d x\right)^{2 / \alpha}=\varepsilon^{-(N-2) / 2}\|U\|_{L^{\alpha}\left(R^{N}\right)}^{2}+O(\varepsilon), \\
\int_{\Omega}\left|\nabla u_{\varepsilon}\right|^{2} d x=\varepsilon^{-(N-2) / 2}\|\nabla U\|_{L^{2}\left(R^{N}\right)}^{2}+O(1), \\
\frac{\int_{\Omega}\left(\left|\nabla u_{\varepsilon}\right|^{2}+a(x)\left|u_{\varepsilon}\right|^{2}\right) d x}{\left(\int_{\Omega}\left|u_{\varepsilon}\right|^{\alpha} d x\right)^{2 / \alpha}}=S+O\left(\varepsilon^{(N-2) / 2}\right),
\end{gathered}
$$

where $U(x)=\left(1+|x|^{2}\right)^{-(N-2) / 2} \in W^{1,2}\left(R^{N}\right)$ is a minimizer of

$$
\left\{\frac{\int_{\Omega}\left(|\nabla u|^{2}+a(x)|u|^{2}\right) d x}{\|u\|_{L^{\alpha}\left(R^{N}\right)}^{2}}\right\}_{u \in W^{1,2}\left(R^{N}\right) \backslash\{0\}} ;
$$

that is,

$$
\begin{aligned}
& \frac{\int_{\Omega}\left(|\nabla U|^{2}+a(x)|U|^{2}\right) d x}{\|U\|_{L^{\alpha}\left(R^{N}\right)}^{2}} \\
& =S=\inf _{u \in W^{1,2}\left(R^{N}\right) \backslash\{0\}} \frac{\int_{\Omega}\left(|\nabla u|^{2}+a(x)|u|^{2}\right) d x}{\|u\|_{L^{\alpha}\left(R^{N}\right)}^{2}} .
\end{aligned}
$$


Set $u_{0}=e_{1} u_{\varepsilon}, v_{0}=e_{2} u_{\varepsilon}$ and $\left(u_{0}, v_{0}\right) \in E$, where $\left(e_{1}, e_{2}\right) \in$ $\left(R^{+}\right)^{2}$ and $\inf _{x \in \bar{\Omega}} F\left(x, e_{1}, e_{2}\right) \geq K$. Then, by (F1), (9), the definition of $S_{F}$, and (69), we obtain that

$$
\begin{aligned}
\sup _{t \geq 0} I & \left(t e_{1} u_{\varepsilon}, t e_{2} u_{\varepsilon}\right) \\
\leq & \frac{N+2}{4 N}\left(\frac{a_{1}\left\|e_{1} u_{\varepsilon}\right\|^{2}+a_{2}\left\|e_{2} u_{\varepsilon}\right\|^{2}}{\left(\int_{\Omega} F\left(x, e_{1} u_{\varepsilon}, e_{2} u_{\varepsilon}\right) d x\right)^{2 / \alpha}}\right)^{2 N /(N+2)} \\
\leq & \frac{N+2}{4 N}\left(\frac{\left(a_{1} e_{1}^{2}+a_{2} e_{2}^{2}\right)\left\|u_{\varepsilon}\right\|^{2}}{\left(\int_{\Omega}\left|u_{\varepsilon}\right|^{\alpha} F\left(x, e_{1}, e_{2}\right) d x\right)^{2 / \alpha}}\right)^{2 N /(N+2)} \\
\leq & \frac{N+2}{4 N}\left(\frac{\left(a_{1} e_{1}^{2}+a_{2} e_{2}^{2}\right)}{K^{2 / \alpha}}\right)^{2 N /(N+2)} \\
& \left.\times\left.\left(\frac{\left\|u_{\varepsilon}\right\|^{2}}{\left(\int_{\Omega}\right.}\right)^{2 N /(N+2)}\right|^{\alpha} d x\right)^{2 / \alpha} \\
\leq & \frac{N+2}{4 N}\left(\frac{\left(a_{1} e_{1}^{2}+a_{2} e_{2}^{2}\right)}{K^{2 / \alpha}}\right)^{2 N /(N+2)} \\
& \times\left(S+O\left(\varepsilon^{(N-2) / 2}\right)\right)^{2 N /(N+2)} \\
\leq & \frac{N+2}{4 N}\left(\frac{\left(a_{1} e_{1}^{2}+a_{2} e_{2}^{2}\right)}{K^{2 / \alpha}}\right)^{2 N /(N+2)} \\
& \times\left(S^{2 N /(N+2)}+O\left(\varepsilon^{(N-2) / 2}\right)\right) \\
& N+2 \\
4 N & S_{F}^{2 N /(N-2)}+O\left(\varepsilon^{(N-2) / 2}\right),
\end{aligned}
$$

where the following fact has been used:

$$
\sup _{t \geq 0}\left(\frac{t^{2}}{2} A-\frac{t^{\alpha}}{\alpha} B\right)=\frac{N+2}{4 N}\left(\frac{A}{B^{2 / \alpha}}\right)^{(N-2) / 2} \quad A, B>0 .
$$

We can choose $\delta_{1}>0$ such that, for all $\varrho_{\lambda, \mu} \in\left(0, \delta_{1}\right)$, we have

$$
c_{\infty}=\left(\frac{1}{4}-\frac{1}{\alpha}\right) S_{F}^{2 N /(N+2)}-\Lambda \varrho_{\lambda, \mu}>0 .
$$

Using the definitions of $J_{\lambda, \mu}$ and $\left(u_{0}, v_{0}\right)$, we get

$$
J_{\lambda, \mu}\left(t u_{0}, t v_{0}\right) \leq \frac{t^{2} \max \left\{a_{1}, a_{2}\right\}}{2}\left\|\left(u_{0}, v_{0}\right)\right\|_{E}^{2} \quad \forall t \geq 0, \lambda, \mu>0,
$$

which implies that there exists $t_{0} \in(0,1)$ satisfying

$$
\sup _{0 \leq t \leq t_{0}} J_{\lambda, \mu}\left(t_{0} u_{0}, t_{0} v_{0}\right)<c_{\infty}, \quad \forall \varrho_{\lambda, \mu} \in\left(0, \delta_{1}\right) .
$$

On the other hand,

$$
\begin{aligned}
\sup _{t \geq t_{0}} J_{\lambda, \mu}\left(t u_{0}, t v_{0}\right) & \\
= & \sup _{t \geq t_{0}}\left(I\left(t u_{0}, t v_{0}\right)+\frac{b_{1}}{4}\left\|t u_{0}\right\|^{4}+\frac{b_{2}}{4}\left\|t v_{0}\right\|^{4}\right. \\
& \left.-\frac{t^{q}}{q} K_{\lambda, \mu}\left(u_{0}, v_{0}\right)\right) \\
\leq & \frac{N+2}{4 N} S_{F}^{2 N /(N-2)}+O\left(\varepsilon^{(N-2) / 2}\right) \\
& +\frac{\max \left\{b_{1}, b_{2}\right\} t^{4}\left(e_{1}^{4}+e_{2}^{4}\right)}{2}\left\|u_{\varepsilon}\right\|^{4} \\
& -\frac{t_{0}^{q}}{q}\left(e_{1}^{q} \lambda b_{0}+e_{2}^{q} \mu c_{0}\right) \int_{B^{N}\left(0, \rho_{0}\right)}\left|u_{\varepsilon}\right|^{q} d x \\
\leq & \frac{N+2}{4 N} S_{F}^{2 N /(N-2)}+O\left(\varepsilon^{(N-2) / 2}\right) \\
& +\frac{\max \left\{b_{1}, b_{2}\right\} t^{4}\left(e_{1}^{4}+e_{2}^{4}\right)}{2}\left\|u_{\varepsilon}\right\|^{4} \\
& -\frac{t_{0}^{q}}{q} m\left(\lambda b_{0}+\mu c_{0}\right) \int_{B^{N}\left(0, \rho_{0}\right)}\left|u_{\varepsilon}\right|^{q} d x
\end{aligned}
$$

where $m=\min \left\{e_{1}^{q}, e_{2}^{q}\right\}, b_{0}=\min b(x)$, and $c_{0}=\min c(x)$ on $B\left(0, \rho_{0}\right)$.

$$
\begin{aligned}
& \text { Let } 0<\varepsilon \leq \rho_{0}^{2} \text {; we get } \\
& \begin{aligned}
\int_{B^{N}\left(0, \rho_{0}\right)}\left|u_{\varepsilon}\right|^{q} d x & =\int_{B^{N}\left(0, \rho_{0}\right)} \frac{1}{\left(\varepsilon+|x|^{2}\right)^{((N-2) / 2) q}} d x \\
& \geq \int_{B^{N}\left(0, \rho_{0}\right)} \frac{1}{\left(2 \rho_{0}^{2}\right)^{((N-2) / 2) q}} d x \\
& =C_{2}\left(N, q, \rho_{0}\right) .
\end{aligned}
\end{aligned}
$$

Combining with (77) and the above inequality, for all $\varepsilon=$ $\varrho_{\lambda, \mu}^{2 /(N-2 p)} \in\left(0, \rho_{0}^{2}\right)$, we have the following.

According to properties of $u_{\varepsilon}$ and $F\left(\left(F_{1}\right)-\left(F_{3}\right), 4.4\right)$, we can conclude that there exists the positive constant $C_{3}$ such that $\left\|u_{\varepsilon}\right\|^{4} \leq C_{3}$, so we have

$$
\begin{aligned}
\sup _{t \geq t_{0}} J_{\lambda, \mu}\left(t u_{0}, t v_{0}\right) & \\
\leq & \frac{N+2}{4 N} S_{F}^{2 N /(N+2)}+O\left(\varrho_{\lambda, \mu}\right) \\
& +\frac{\max \left\{b_{1}, b_{2}\right\} t^{4}\left(e_{1}^{4}+e_{2}^{4}\right)}{2} C_{3}-\frac{t_{0}^{q}}{q} \varrho_{\lambda, \mu} m C_{2} .
\end{aligned}
$$

There exists a constant $\Lambda_{1}$, such that $((N+2) / 4 N) S_{F}^{2 N /(N+2)}$ $\Lambda_{1} \varrho_{\lambda, \mu} \leq c_{\infty}$. 
Hence, we can choose $\delta_{2}>0$ such that, for all $\varrho_{\lambda, \mu} \in$ $\left(0, \delta_{2}\right)$, we obtain

$$
\begin{aligned}
& O\left(\varrho_{\lambda, \mu}\right)+\frac{\max \left\{b_{1}, b_{2}\right\} t^{4}\left(e_{1}^{4}+e_{2}^{4}\right)}{2} C_{3}-\frac{t_{0}^{q}}{q} \varrho_{\lambda, \mu} m C_{2} \\
& <-\Lambda_{1} \varrho_{\lambda, \mu} .
\end{aligned}
$$

If we see $C^{*}=\min \left\{\delta_{1}, \rho_{0}^{N-2}, \delta_{2}\right\}$ and $\varepsilon=\varrho_{\lambda, \mu}^{2 /(N-2 p)}$, then for $\lambda \in\left(0, C^{*}\right)$ we have

$$
\sup _{t \geq t_{0}} J_{\lambda, \mu}\left(t u_{0}, t v_{0}\right)<c_{\infty}
$$

Finally, we prove that $\theta_{\lambda, \mu}^{-}<c_{\infty}$ for all $\varrho_{\lambda, \mu} \in\left(0, C^{*}\right)$. Recall that $\left(u_{0}, v_{0}\right)=\left(e_{1} u_{\varepsilon}, e_{2} u_{\varepsilon}\right)$. It is easy to see that

$$
\int F\left(x, u_{0}, v_{0}\right) d x>0
$$

Combining this with Lemma 9, from the definition of $\theta_{\lambda, \mu}^{-}$ and (81), we get that there exists $t_{0}>0$ such that $\left(t_{0} u_{0}, t_{0} v_{0}\right) \in$ $N_{\lambda, \mu}^{-}$and

$$
\theta_{\lambda, \mu}^{-} \leq J_{\lambda, \mu}\left(t_{0} u_{0}, t_{0} v_{0}\right) \leq \sup _{t \geq 0} J_{\lambda, \mu}\left(t_{0} u_{0}, t_{0} v_{0}\right)<c_{\infty}
$$

for all $\varrho_{\lambda, \mu} \in\left(0, C^{*}\right)$.

Theorem 17. If $\varrho_{\lambda, \mu} \in\left(0, C_{0}^{*}\right)$ and (F1)-(F3) hold, then $J_{\lambda, \mu}$ has a minimizer $\left(u_{0}^{-}, v_{0}^{-}\right)$in $N_{\lambda, \mu}^{-}$and it satisfies the following:

(i) $J_{\lambda, \mu}\left(u_{0}^{-}, v_{0}^{-}\right)=\theta_{\lambda, \mu}^{-}$,

(ii) $\left(u_{0}^{-}, v_{0}^{-}\right)$is a positive solution of problem (1),

where $C_{0}^{*}=\min \left\{C^{*}, C_{0}\right\}$.

Proof. By Lemma 10(ii), there is a $(P S)_{\theta_{\lambda, \mu}^{-}}$-sequence $\left\{\left(u_{n}\right.\right.$, $\left.\left.v_{n}\right)\right\} \subseteq N_{\lambda, \mu}^{-}$in $E$ for $J_{\lambda, \mu}$ for all $\varrho_{\lambda, \mu} \in\left(0, C_{0}\right)$. From Lemmas 15, 16 , and 8 (ii), for $\varrho_{\lambda, \mu} \in\left(0, C^{*}\right), J_{\lambda, \mu}$ satisfies $(P S)_{\theta_{\lambda, \mu}^{-}}$condition and $\theta_{\lambda, \mu}^{-}>0$. Since $J_{\lambda, \mu}$ is coercive on $N_{\lambda, \mu}$, we get that $\left(u_{n}, v_{n}\right)$ is bounded in $E$. Therefor, there exists a subsequence still denoted by $\left(u_{n}, v_{n}\right)$ and $\left(u_{0}^{-}, v_{0}^{-}\right) \in N_{\lambda, \mu}^{-}$such that $\left(u_{n}, v_{n}\right) \rightarrow$ $\left(u_{0}^{-}, v_{0}^{-}\right)$strongly in $E$ and $J_{\lambda, \mu}\left(u_{0}^{-}, v_{0}^{-}\right)=\theta_{\lambda, \mu}^{-}>0$ for all $\varrho_{\lambda, \mu} \in\left(0, C_{0}^{*}\right)$. Finally, by the same arguments as in the proof of Theorem 11, for all $\varrho_{\lambda, \mu} \in\left(0, C_{0}^{*}\right)$, we have that $u_{0}^{-}$is a positive solution of problem (1).

Now, we complete the proof of Theorems 1 and 2. By Theorem 11, we obtain that, for all $\varrho_{\lambda, \mu} \in C\left(q, N, K, S,|\Omega|, a_{1}\right.$, $a_{2}$ ), problem (1) has a positive solution $\left(u_{0}^{+}, v_{0}^{+}\right) \in N_{\lambda, \mu}^{+}$. On the other hand, from Theorem 17, we get the second positive solution $\left(u_{0}^{-}, v_{0}^{-}\right) \in N_{\lambda, \mu}^{-}$for all $0<\varrho_{\lambda, \mu}<C_{0}^{*}<C(q, N$, $\left.K, S,|\Omega|, a_{1}, a_{2}\right)$. Since $N_{\lambda, \mu}^{+} \cap N_{\lambda, \mu}^{-}=\emptyset$, this implies that $\left(u_{0}^{+}, v_{0}^{+}\right)$and $\left(u_{0}^{-}, v_{0}^{-}\right)$are distinct. This completes the proof of Theorems 1 and 2 .

\section{Conflict of Interests}

The authors declare that there is no conflict of interests regarding the publication of this paper.

\section{References}

[1] G. R. Kirchhoff, Vorlesungen über mathematische Physik: Mechanik, B.G. Teubner, Leipzig, Germany, 1883.

[2] F. Julio, S. A. Correa, and G. M. Figueiredo, "On an elliptic equation of p-Kirchhoff type via variational methods," Bulletin of the Australian Mathematical Society, vol. 74, pp. 246-277, 2006.

[3] C. Y. Chen, Y. C. Kuo, and T. F. Wu, “The Nehari manifold for a Kirchhoff type problem involving sign-changing weight functions," Journal of Differential Equations, vol. 250, no. 4, pp. 1876-1908, 2011.

[4] J. H. Jin and X. Wu, "Infinitly many radial solutions for Kirchhoff type problem in $\mathbb{R}^{N}$," Journal of Mathematical Analysis and Applications, vol. 369, no. 2, pp. 564-574, 2010.

[5] X. M. He and W. M. Zou, "Infinitely many positive solutions for Kirchhoff-type problems," Nonlinear Analysis, Theory, Methods and Applications, vol. 70, no. 3, pp. 1407-1414, 2009.

[6] K. Perera and Z. T. Zhang, "Nontrivial solutions of Kirchhofftype problems via the Yang index," Journal of Differential Equations, vol. 221, no. 1, pp. 246-255, 2006.

[7] M. Ferrara, S. Khademloo, and S. Heidarkhani, "Multiplicity results for perturbed fourth-order Kirchhoff type elliptic problems," Applied Mathematics and Computation, vol. 234, pp. 316325, 2014

[8] F. Julio, S. A. Correa, and R. G. Nascimento, "On a nonlocal elliptic system of $p$-Kirchhoff-type under Neumann boundary condition," Mathematical and Computer Modelling, vol. 49, no. 3-4, pp. 598-604, 2009.

[9] M. Willem, Minimax Theorems, Birkhäuser, Boston, Mass, USA, 1996.

[10] Y. Shen and J. Zhang, "Multiplicity of positive solutions for a semilinear $p$-Laplacian system with Sobolev critical exponent," Nonlinear Analysis. Theory, Methods \& Applications, vol. 74, no. 4, pp. 1019-1030, 2011.

[11] K. J. Brown and T.-F. Wu, "A semilinear elliptic system involving nonlinear boundary condition and sign-changing weight function," Journal of Mathematical Analysis and Applications, vol. 337, no. 2, pp. 1326-1336, 2008.

[12] T. F. Wu, "A semilinear elliptic equations involving nonlinear bou ndary codition and signchanging potential," Electronic Journal of Differential Equations, vol. 131, pp. 1-15, 2006.

[13] H. Brézis and A. Lieb, "A relation between pointwise convergence of functions and convergence of functionals," Proceedings of the American Mathematical Society, vol. 88, pp. 486-490, 1983.

[14] T.-S. Hsu, "Multiple positive solutions for a critical quasilinear elliptic system with concave-convex nonlinearities," Nonlinear Analysis: Theory, Methods \& Applications, vol. 71, no. 7-8, pp. 2688-2698, 2009. 


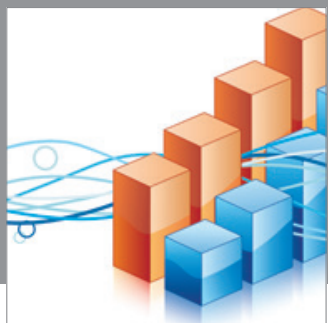

Advances in

Operations Research

mansans

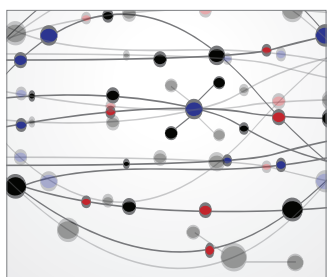

The Scientific World Journal
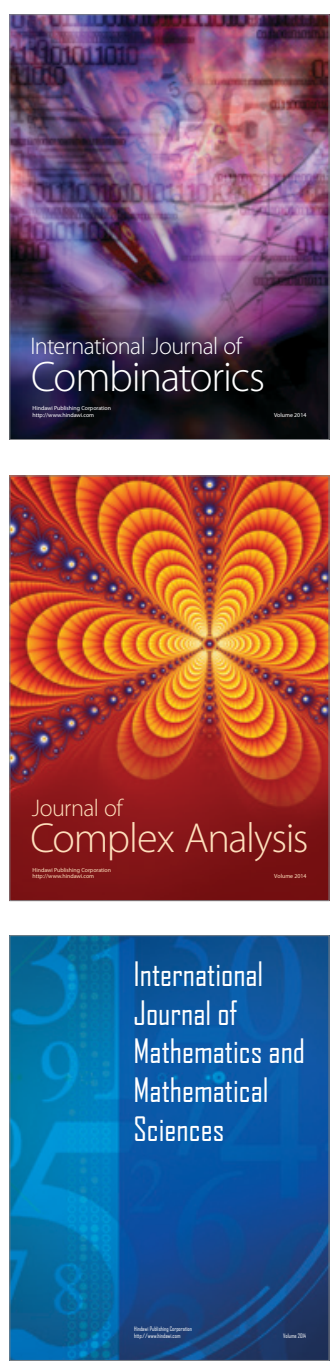
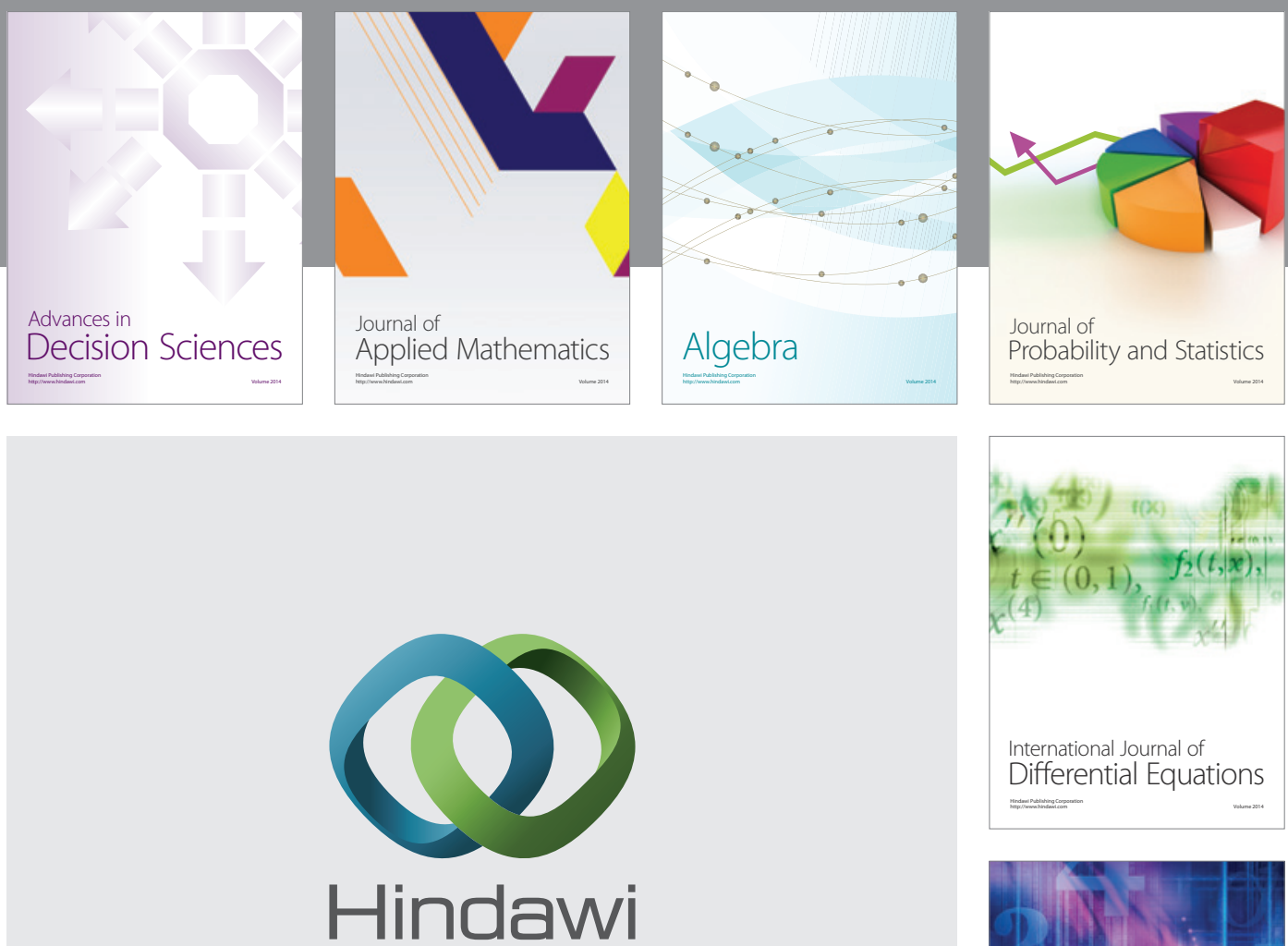

Submit your manuscripts at http://www.hindawi.com
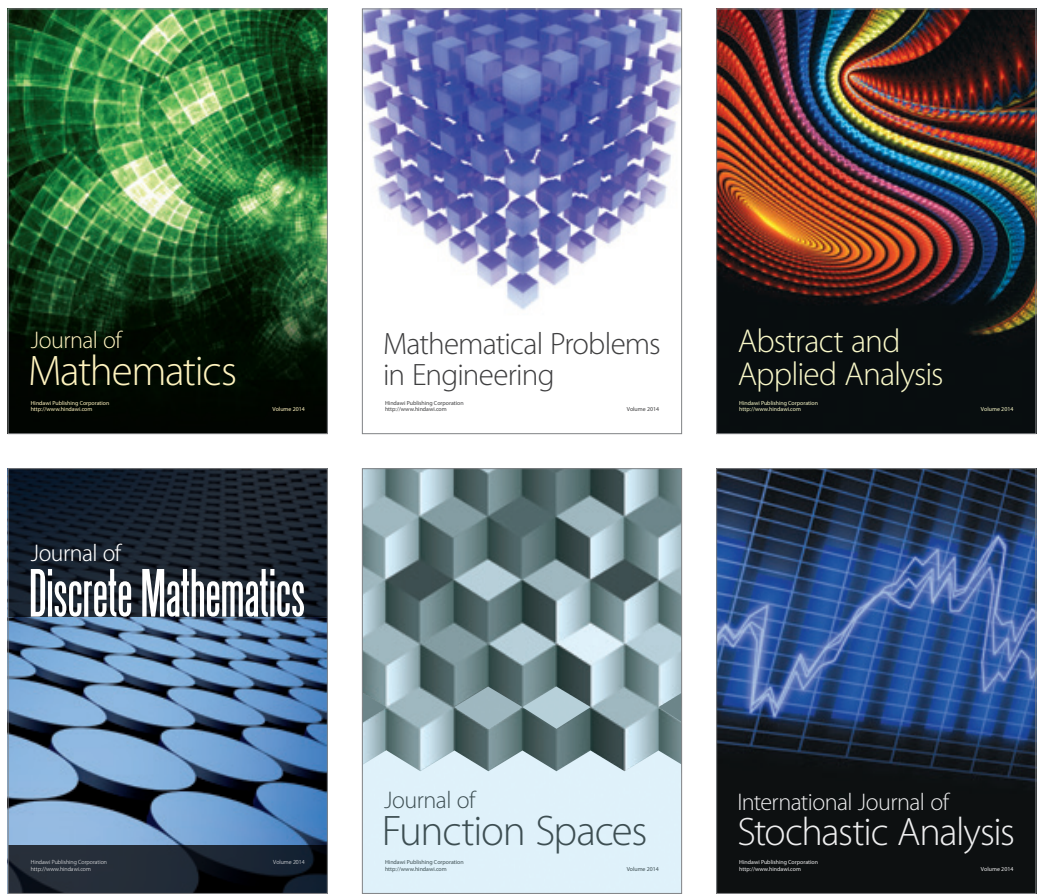

Journal of

Function Spaces

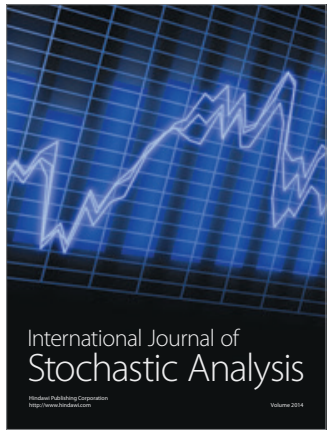

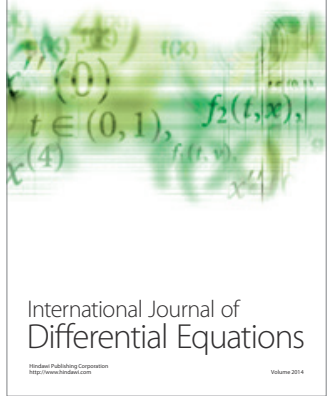
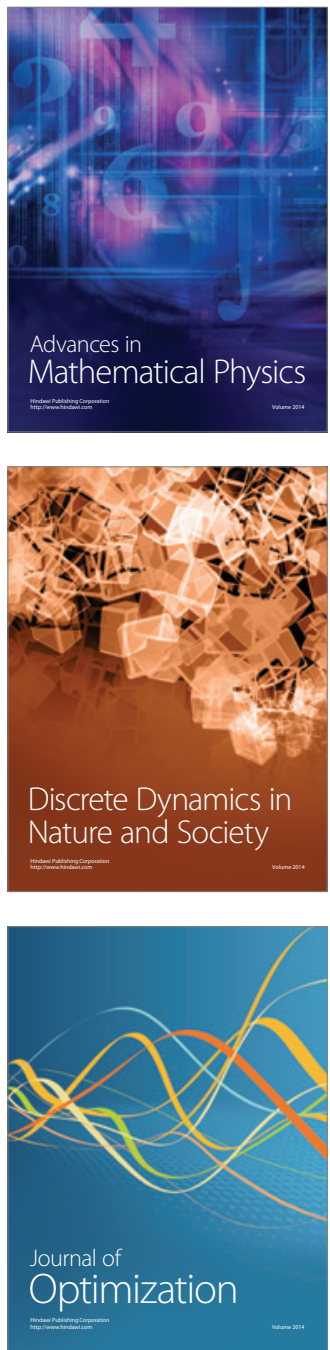